\title{
Agencias de comunicación en España en la encrucijada digital. Prácticas, perspectivas y visión de futuro
}

\section{Communication agencies at the digital crossroads in Spain. Practices, perspectives and vision of future}

\author{
Carmen Costa-Sánchez; Miguel Túñez-López; María-Isabel Míguez-González
}

Cómo citar este artículo:

Costa-Sánchez, Carmen; Túñez-López, Miguel; Míguez-González, María-Isabel (2019). “Agencias de comunicación en España en la encrucijada digital. Prácticas, perspectivas y visión de futuro". El profesional de la información, v. 28, n. 6, e280606.

https://doi.org/10.3145/epi.2019.nov.06

Artículo recibido el 25-03-2019

Aceptación definitiva: 14-09-2019

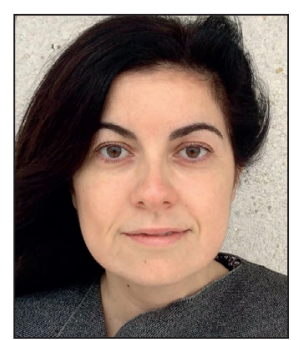

Carmen Costa-Sánchez $\bowtie$ https://orcid.org/0000-0001-8154-9537

Universidade da Coruña Facultade de Ciencias da Comunicación Campus de Elviña, s/n. 15001 A Coruña, España carmen.costa@udc.es

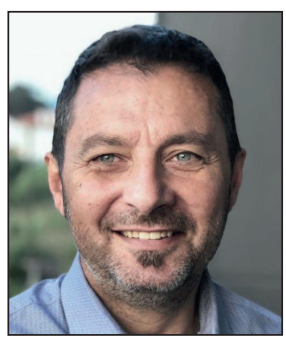
Miguel Túñez-López https://orcid.org/0000-0002-5036-9143

Universidade de Santiago de Compostela

Facultade de Ciencias da Comunicación Av. Castelao, s/n. 15706 Santiago de Compostela, España miguel.tunez@usc.es

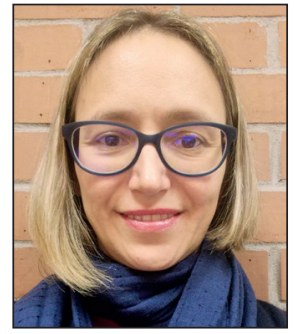

María-Isabel Míguez-González http://orcid.org/0000-0002-0580-8493

Universidade de Vigo

Facultade de Ciencias Sociais e da Comunicación

Campus A Xunqueira, s/n. 36005

Pontevedra, España

mabelm@uvigo.es

\section{Resumen}

El estudio analiza los principales retos percibidos por el sector de la consultoría de comunicación en España en un contexto de post-convergencia. Como metodología se emplea el cuestionario en profundidad a profesionales de la comunicación de agencia y el análisis de contenido de sus webs corporativas y plataformas sociales. Los resultados apuntan a una situación en transición entre viejas y nuevas prácticas, en la que la digitalización es percibida como una necesidad actual y un reto a su capacidad de adaptación, al tiempo que sólo algunas agencias utilizan los nuevos canales que ofrece la Red para su propia gestión de la transparencia y visibilidad online. Se detecta una doble reactividad: con respecto a su visión de futuro y a su liderazgo de marca digital. La investigación aporta claves que pueden ayudar a académicos, docentes y profesionales a evaluar el sector y a detectar necesidades de adaptación de presente y de futuro.

\section{Palabras clave}

Agencias de comunicación; Consultoría; Comunicación; Ecosistema digital; Comunicación corporativa; Relaciones públicas; Comunicación digital; Marketing digital; Medios sociales; Profesión; Profesionales; Práctica profesional; Convergencia mediática; España.

\section{Abstract}

The study analyses the main challenges perceived by communication consulting sector in Spain in a post-convergence context. An in-depth questionnaire to communication agency professionals and content analysis of their corporate websites and social platforms was used as methodology. The results point to a situation in transition between old and new practices, in which digitalisation is perceived as a current need and a challenge to sector's adaptation capacity, while only 
some agencies use the new channels offered by Internet for their own management of transparency and online visibility. A double reactivity is detected: with respect to their vision of the future, and to digital brand leadership. The research provides clues that can help researchers, professors and professionals to evaluate the sector and detect adaptation needs for the present and the future.

\section{Keywords}

Communication agencies; Consulting; Communication; Digital ecosystem; Corporate communication; Public relations; Digital communication; Digital marketing; Social media; Profession; Professionals; Professional practice; Media convergence; Spain.

\section{Introducción}

El estudio analiza los principales retos percibidos por el sector de la consultoría de comunicación en España en un contexto de post-convergencia.

Según Röttger y Preusse (2013), la consultoría se entiende, desde el punto de vista del servicio profesional, como una actividad orientada a resolver problemas y a optimizar la capacidad de los clientes para actuar. En el contexto específico de la comunicación estratégica, el término se emplea para designar a los proveedores de servicios externos, ya sean consultores individuales o agencias.

Pero la confusión terminológica que se acusa en gestión de comunicación afecta también a la denominación que reciben las empresas que ofertan servicios de comunicación, comunicación estratégica y relaciones públicas (Rodríguez-Salcedo; Buil-Gazol, 2015; Buil-Gazol; Rodríguez-Salcedo, 2017). Urzáiz-Fernández-Del-Castillo (1997) utiliza el nombre de agencias de relaciones públicas para referirse a aquellas organizaciones con forma jurídica de sociedad mercantil, que con el objeto social único y adecuado, el personal técnico especializado necesario y los medios suficientes, se dedican profesionalmente a prestar servicios a terceros, en materia de comunicación social integral de cara a lograr una mejor imagen pública y a la consecución de sus fines empresariales e institucionales. Rojas-Orduña (2008) define este perfil empresarial como una empresa que no sólo ofrece consultoría estratégica sobre comunicación, tanto a nivel interno como externo, sino que también puede y suele ser la encargada de llevar a cabo el programa que ofrece a sus clientes por unos honorarios determinados. La gran diferencia entre la dirección/gabinete de comunicación y las agencias de comunicación radica en que éstas son independientes, mientras que el gabinete suele pertenecer a la organización, más allá de que algunas de estas asesorías puedan autodenominarse gabinetes (Almansa-Martínez, 2005).

Aunque cada opción tiene sus ventajas e inconvenientes y se trata de opciones habitualmente complementarias (Miquel-Segarra; López-Font; Gil-Soldevila, 2018), la contratación de una consultora externa, según Rojas-Orduña (2008), aporta ventajas como la reducción de costes tanto de producción como de mantenimiento, dada la facilidad para finalizar la relación cuando los servicios no son necesarios, o la especialización.

Según Frandsen, Johansen y Pang (2013), las agencias o consultoras externas desempeñan un papel importante cuando las organizaciones públicas o privadas necesitan usar la comunicación para alcanzar sus metas. Sin embargo, teniendo en cuenta el tamaño e impacto de este sector, resulta llamativo el escaso interés académico que se ha prestado a este tipo de consultoras desde las ciencias de la comunicación y de la investigación en relaciones públicas (Frandsen; Johansen; Pang, 2013; Röttger; Preusse, 2013; Von-Platen, 2016).

El presente trabajo realiza un acercamiento a las prácticas y percepciones de las consultoras de mayor facturación en España para conocer la situación mainstream en un contexto de convergencia mediática (Jenkins, 2008) marcado por la importancia de la comunicación online multiplataforma. El acercamiento a la percepción de los profesionales de agencia sobre la situación del mercado español, así como la profundización en sus propias estrategias de marca permitirá continuar una línea de investigación reciente para conocer en profundidad la visión y los retos que están afrontando en la encrucijada digital.

\subsection{El sector de las agencias de comunicación en España}

Desde que en 1960 se creara la primera empresa española de relaciones públicas (SAE de Relaciones Públicas) con el objetivo de generar aceptación y prestigio hacia diferentes públicos (Barquero-Cabrero, 1999), mucho ha cambiado la situación de la consultoría de comunicación en España. La transición política trajo consigo la acuciante necesidad de comunicación para las organizaciones (Ramírez, 1995), lo que tuvo como consecuencia una etapa de expansión y paulatina profesionalización de los gabinetes de comunicación (Almansa-Martínez, 2004). Como indicador de madurez en el sector puede citarse la creación en 1991 de la Asociación Española de Empresas Consultoras en Comunicación y Relaciones Públicas (Adecec), justo un año antes de la creación de la Asociación de Directivos de la Comunicación (Dircom). Martín-Martín (1998) dibujaba a finales de los 90 un panorama cuantitativamente destacado en nuestro país: 3.000 directores de comunicación en el sector empresarial y de la administración, 184 asesorías de comunicación, 3.500 profesionales trabajando en gabinetes de comunicación y 1.600 revistas informativas especializadas. 
Según recoge $\operatorname{Adecec}(\mathrm{s} / \mathrm{f}$ ) el sector de las agencias de comunicación en España es muy amplio. En cuanto al tipo de servicio que ofrecen, existen agencias especializadas en servicios de comunicación específicos, y otras que ofrecen servicios integrales de comunicación. El tamaño de las agencias también es muy diverso y existen desde profesionales freelance, a agencias locales, nacionales y multinacionales (pertenecientes a una red de oficinas en numerosos países) que además pueden estar especializadas en un sector concreto, las llamas agencias boutique.

En la actualidad describen el sector de la consultoría de comunicación en España dos tendencias: la concentración empresarial, en un intermitente contexto de fusiones y adquisiciones, y la consecuente concentración del mercado. En efecto, a pesar del elevado número de pequeñas consultoras que aparecen y desaparecen, el 50\% de la facturación total declarada en el registro mercantil lo acumulan poco más de las 50 primeras empresas por volumen y el top 10 de empresas por volumen de negocio acapararía casi 90 de los 378 millones de euros controlados (Scopen, 2017). Desde un punto de vista más cualitativo a partir de datos cuantificados (Scopen, 2017), se detecta un elevado nivel de satisfacción con el trabajo que realizan (un 4,24 sobre 5), además de un modelo de relación habitual y estable, en lugar de por proyectos, de 4,53 años de relación media cliente-consultora. Las consultoras en España son valoradas en relación a su capacidad de innovación y creatividad, el conocimiento del mercado y de la marca, su proactividad y su capacidad relacional. Específicamente en comunicación digital se valora su capacidad de creación de contenidos y los servicios de asesoramiento estratégico y analítica o medición.

En cuanto al capital humano, según los datos del estudio de Miquel-Segarra, López-Font y Gil-Soldevila (2018) las consultoras españolas cuentan con un elevado porcentaje de profesionales que son titulados en Periodismo (en torno a un 60\%), seguido de titulados en Publicidad y Relaciones Públicas (un 21\%), con mayor presencia de mujeres que de hombres. Se trata de un personal joven cuyas características son similares a las del perfil general del dircom descrito por Martín-Llaguno, Miquel-Segarra y Navarro-Beltrá (2015).

\subsection{Comunicación de agencia en un entorno post-convergente}

Si en el siglo XX la consultoría de comunicación consistía fundamentalmente en la gestión de medios y de la reputación, el advenimiento de las nuevas tecnologías desafía la conceptualización tradicional de este rol. Los consultores necesitan rediseñar sus procesos de trabajo y pueden tener un papel crucial traduciendo las demandas de los stakeholders y gestionando la transparencia, las relaciones y la reputación en un entorno online complejo y cambiante (Von-Platen, 2016).

En los inicios del siglo XXI los servicios de relaciones con los medios (o de prensa) suponían el $80 \%$ del volumen de honorarios de las asesorías de relaciones públicas (Almansa-Martínez, 2005) y uno de sus servicios más demandados (Adecec, 2008). Sin embargo diez años después, tras la eclosión de la web 2.0, la comunicación móvil y los medios sociales, las agencias de comunicación afrontan nuevas necesidades de comunicación vinculadas a la influencia 2.0, la reputación online, la creación y combinación de estrategias tradicionales y online, y la generación de contenidos para los nuevos canales (Aced-Toledano, 2013). Los profesionales valoran positivamente la importancia de los medios sociales en su práctica profesional (Alikilic; Atabek, 2012; Triantafillidou; Yannas, 2014). Estudios recientes han detectado el progresivo declive de las relaciones informativas como buque insignia de su cartera de servicios en favor de los servicios de comunicación digital (Buil-Gazol; Rodríguez-Salcedo, 2017; Míguez-González; Costa-Sánchez; Túñez-López, 2019). A este respecto, la situación de este tejido empresarial en Europa parece polarizada:

“Hay una creciente división entre las agencias que están modernizando su oferta para satisfacer las necesidades cambiantes de los clientes y las presiones externas, y las que no lo están. No se puede negar que las agencias que adquieren nuevas habilidades digitales especializadas y creativas están superando a las que no las tienen" (Hutton, 2017, p. 9).

La necesidad de las organizaciones de comunicarse en el entorno 2.0 ha conllevado la creación de departamentos especializados en redes sociales y comunicación digital. En esta área compiten directamente con agencias de publicidad o de marketing online y con agencias especializadas en comunicación en nuevos medios (Estanyol-Casals; Lalueza-Bosch, 2014; Von-Platen, 2016). Esto es patente en el contexto internacional: Neil y Schauster (2018) destacan que los profesionales de las relaciones públicas están ofreciendo a sus clientes experiencias de tipo publicitario como posts patrocinados en medios sociales, publicidad nativa o marketing de influencers, mientras que las agencias de publicidad ofertan servicios de gestión de comunidades online y analítica. En esta misma idea incide el USC Annenberg Center for Public Relations (2019) que constata la competencia entre agencias de relaciones públicas, agencias de publicidad, empresas dedicadas exclusivamente al mundo digital e incluso consultorías de gestión, todas ellas, según el estudio, con profundas raíces tecnológicas.

El informe del USC Annenberg Center for Public Relations (2019) destaca que más de un 80\% de los ejecutivos de agencias de comunicación esperan cambios considerables o drásticos en la industria de las relaciones públicas en los próximos cinco años y más del $80 \%$ de los comunicadores afirman que las innovaciones tecnológicas dirigirán este cambio. Señalan YouTube, Instagram, Google, LinkedIn, Twitter y Facebook como las plataformas que los profesionales consideran más relevantes para alcanzar la efectividad de las estrategias comunicativas a cinco años vista y destrezas relacionadas con el análisis de datos masivos (big data), producción de vídeo, posicionamiento en buscadores, diseño digital y generación de experiencias de usuarios, como las más necesarias en el futuro. 
En este contexto, Von-Platen (2016) incide en que a medida que las nuevas tecnologías de comunicación se integran en las funciones centrales de la organización, los consultores de comunicación ven cómo cambia su rol de ser un proveedor de servicios periféricos de comunicación al de desarrollador de procesos empresariales centrales, lo que puede incrementar considerablemente el valor de su función. En cualquier caso se detecta una desestabilización de las estructuras y roles tradicionales que tiene consecuencias para la profesión y si la comunicación estratégica aspira a ser relevante en el mundo digital debe explorar cómo crear y mantener sus nuevos roles.

Teniendo en cuenta estos elementos, el presente estudio pone el foco en la adaptación de los servicios de agencia al ecosistema mediático actual (Scolari, 2015) marcado por la convergencia (Salaverría, 2003) o post-convergencia (Ramírez-Sánchez, 2018) y en su percepción sobre la futura evolución de las tendencias de la comunicación (Zerfass et al., 2017; 2018).

\section{Metodología}

El sector empresarial de la consultoría de comunicación y relaciones públicas en España abarca un conjunto de entidades y de iniciativas difíciles de acotar y de cuantificar. Miquel-Segarra, López-Font y Gil-Soldevila (2018) explican que podría estar entre las 500 (tomando como referencia el código CNAE 7021) o las más de 1.000 según el informe elaborado por Torres \& Carrera (2011). En la presente investigación se parte del censo de consultoras de comunicación por facturación (PRNoticias; Torres \& Carrera, 2018), dado que interesa la situación de las entidades que lideran el mercado en España y que, por tanto, trabajan para las empresas y organizaciones más influyentes, y a su vez disponen de recursos y equipos suficientes para afrontar los nuevos retos y demandas del sector.

A partir de dicho escenario, se realizó un trabajo de actualización de las empresas conformantes de dicho ranking a raíz de los consiguientes movimientos empresariales acontecidos entre 2018 y 2019. Finalmente el universo de estudio estuvo compuesto por las 40 empresas conformantes de dicho ranking, de las que 31 participaron en el análisis, estando representadas las 20 primeras firmas que encabezan la facturación en España y, por lo tanto, las empresas que concentran más de la mitad del mercado español de consultoría de comunicación.

Tabla 1. Universo de estudio. Consultoras con mayor facturación en España. Las 31 que están con fondo sombreado son las que participaron en el análisis.

\begin{tabular}{|c|c|c|c|}
\hline 1 & Llorente y Cuenca & 2 & Atrevía \\
\hline 3 & Globally' & 4 & Ketchum ${ }^{2}$ \\
\hline 5 & Kreab & 6 & $B C W^{3}$ \\
\hline 7 & Equipo Singular & 8 & Estudio de Comunicación \\
\hline 9 & Apple Tree & 10 & Tinkle Consultants \\
\hline 11 & Edelman & 12 & Marco de Comunicación \\
\hline 13 & Román y Asociados & 14 & Weber Shandwick España ${ }^{4}$ \\
\hline 15 & Porter Novelli & 16 & Interbrand Branding ${ }^{5}$ \\
\hline 17 & Evercom & 18 & Hill \& Knowlton \\
\hline 19 & Coonic & 20 & Nota Bene \\
\hline 21 & Ogilvy Public Relations & 22 & Neolabels \\
\hline 23 & Ogilvy \& Mather Publicidad & 24 & Planner Media \\
\hline 25 & Ulled y Asociados & 26 & Torres \& Carrera \\
\hline 27 & Asesores PR y Comunicación & 28 & Solsona Comunicación \\
\hline 29 & Berbés Asociados & 30 & Lina Ortas Comunicación \\
\hline 31 & Text 100 & 32 & Cariotipo $\mathrm{MH} 5$ \\
\hline 33 & ACHCambre & 34 & Grayling \\
\hline 35 & Eurocofin & 36 & Idex \\
\hline 37 & Best Relations & 38 & Mas Consulting \\
\hline 39 & Deva Comunicación Financiera & 40 & MBN Comunicación \\
\hline
\end{tabular}

A continuación se diseñó un cuestionario online semiestructurado y autoadministrado compuesto por diversos tipos de preguntas: dicotómicas, de elección única, de elección múltiple, de escala y preguntas abiertas. El cuestionario se envió por correo electrónico y se recibieron las respuestas entre el 10 de noviembre de 2018 y el 25 de febrero de 2019 . Los principales bloques temáticos del cuestionario estuvieron conformados por:

1. Datos del profesional contactado (nombre, edad, empresa, datos de contacto);

2. Principales magnitudes de la agencia (año de fundación/ establecimiento en España, actividad en otros países, especialización);

3. Características del equipo de profesionales de la consultora (número de profesionales, sexo, media de edad, titulación más habitual, formación de posgrado); 
4. Departamentos de comunicación online y audiovisual (presencia, denominación y funciones);

5. Portafolio de actividades y servicios y percepción del nivel estratégico de los servicios ofertados (los principales, los que han perdido relevancia, los que han ganado relevancia);

6. Tendencias en comunicación (de futuro inmediato, en las que se encuentran trabajando, conocimientos especializados de futuro) $)^{6}$

7. Principales retos percibidos;

8. Necesidades de formación (tiempo dedicado, áreas en las que le gustaría mejorar).

Los participantes en el estudio ocupan en su mayoría puestos en altos niveles en la estructura de la agencia, lo que les da una visión general del mercado de la consultoría de comunicación en España y un elevado conocimiento de su propia organización a todos los niveles. Se describen a continuación sus perfiles y género:

- Presidente / vicepresidente: Burson Cohn \& Wolfe (M), Torres \& Carrera (M), Deva (H).

- Consejero delegado: $M B N(\mathrm{H})$.

- CEO / director general / de agencia: Edelman $(\mathrm{H})$, Hill + Knowlton $(\mathrm{H})$, Coonic $(\mathrm{H})$, Apple Tree (M), Atrevia (M), Tinkle Consultants (M), Planner media (M), Asesores de Relaciones Públicas (H), Lina Ortas Comunicación (M), NewLink (H), Equipo Singular (H), Grupo Idex (H), Mas Consulting, Ogilvy (M).

- Director de marketing y comunicación: Kreab (H), Marco de Comunicación (H), Interbrand (M).

- Desarrollo de negocio: Evercom (H).

- Director de comunicación financiera: Román y Asociados (H).

- Director de área de formación: Estudio de comunicación (H), OmniCom PR (M).

- Director de estrategia: Best (H).

- Director de cuentas: Nota Bene (M), Weber Shandwick (M), ACH Cambre (M).

- Senior HR: Llorente \& Cuenca (M).

En una segunda etapa de la investigación se llevó a cabo un análisis de las webs corporativas y redes sociales de las agencias de comunicación del universo de estudio (las 40 de más facturación en España) con el objetivo de establecer el grado de desarrollo que plantean en su propia comunicación online en torno a tres ejes:

1) Presencia de la firma en internet y redes sociales;

2) Identificación y presencia de sus profesionales en las redes;

3) Desarrollo de su propia marca digital por medio de iniciativas que ayuden a posicionar la agencia como innovadora y atenta a los cambios del entorno digital.

Se construyó un índice numérico que puntuaba diversos elementos de los tres bloques según se describe en la tabla 2. Este medidor, denominado Índice de liderazgo digital, ha permitido diferenciar entre agencias con mayor o menor desarrollo de liderazgo en la Red, aproximarse a la situación general del sector y correlacionar las características de la agencia (tamaño según el número de empleados y tipo de agencia atendiendo a su ámbito de actuación) con la puntuación obtenida en dicho indicador.

Además, permite contraponer la visión de los profesionales con respecto al contexto online y de continuo cambio (primera parte del estudio) y el desarrollo estratégico de su propia comunicación digital (segunda parte).

Tabla 2. Índice de liderazgo digital

\begin{tabular}{|c|c|c|}
\hline Bloque & Items de análisis & Puntuación \\
\hline \multirow{3}{*}{ 1. La agencia en internet } & a. Web corporativa local & 1 punto \\
\hline & $\begin{array}{l}\text { b. Redes sociales } \\
\text { b.1. Superior a la media } \\
\text { b.2. Inferior a la media } \\
\text { b.3. Ninguna }\end{array}$ & $\begin{array}{l}\text { b.1. } 2 \text { puntos } \\
\text { b.2. } 1 \text { punto } \\
\text { b.3. } 0 \text { puntos }\end{array}$ \\
\hline & $\begin{array}{l}\text { c. Espacio de noticias / actualidad de la } \\
\text { agencia }\end{array}$ & 1 punto \\
\hline \multirow{4}{*}{ 2. Los profesionales de la agencia en internet } & d. Identificados & 1 punto \\
\hline & e. E-mail o teléfono & 1 punto \\
\hline & f. En redes & 2 puntos \\
\hline & g. Con web personal & 3 puntos \\
\hline 3. Las iniciativas de la agencia en internet & $\begin{array}{l}\text { h. Observatorio } \\
\text { i. Revista online } \\
\text { j. Podcasting } \\
\text { k. Aplicación móvil } \\
\text {... }\end{array}$ & 1 punto/ cada una \\
\hline Total (máximo) & & $11-\ldots$ \\
\hline
\end{tabular}




\section{Resultados}

\subsection{Perfil de la agencia de comunicación en España}

Las principales agencias de comunicación operantes en España se fundaron en los años 90, por lo que se trata de empresas consolidadas con una trayectoria superior a los 20 años. La mayoría ofrecen servicios en otros países, bien porque pertenecen a grupos de comunicación multinacionales y su presencia en España es una franquicia, bien porque se trata de empresas locales (nacidas en el mercado español) que han ampliado cartera y servicios hacia otros países, destacando Portugal y países de América Latina aprovechando la ventaja idiomática.

En su mayoría se trata de empresas medianas, atendiendo al número de profesionales que trabajan en ellas, que se encuentra entre 51 y 100 (en un 42\% de los casos) o entre 26 y 50 (pequeña empresa, en un 19\%).

La mayoría de dichos profesionales son mujeres en una proporción que oscila entre el 30(H)/70(M) (38,7\%) y 40(H)/60(M) $(25,8 \%)$. Se trata además de profesionales jóvenes (de 31 a 34 años) en un $53 \%$ de los casos, seguidos por un grupo de edad ligeramente superior (de 35 a 39 años) en un 27\%. La titulación mayoritaria de sus profesionales es Periodismo (87\%), por delante de Publicidad y Relaciones públicas (58\%), Relaciones Públicas (38,7\%), Publicidad (19,4\%) o Comunicación audiovisual $(19,4 \%)$. La formación de posgrado que más demandan en la actualidad es la de máster o curso de posgrado en Comunicación digital (76\%) o máster o curso de posgrado en Marketing digital (48\%), lo que supone un indicador de la preferencia actual por perfiles especializados en las plataformas, los lenguajes y el contexto de la digitalización.

La formación de posgrado que más demandan es la de máster y curso de posgrado en Comunicación digital

La mayoría son agencias generalistas, que prestan servicios integrales para todo tipo de sectores (63\%), aunque algunas destacan su especialización en sectores específicos (en concreto: turismo, salud, lifestyle, belleza, consumo, comunicación financiera y tecnología) o tipos de actuación (comunicación de crisis, gestión de marcas, gabinete de prensa ylas organización de eventos, como los más destacados).

\subsection{Percepciones de los profesionales: tendencias y grado de madurez del sector}

Los profesionales de las agencias participantes en el estudio fueron requeridos sobre los retos y el futuro que se le plantea a la comunicación y a la actividad que desarrollan. En una estrategia bottom-up pueden identificarse las reflexiones de interés con un grado de acuerdo coincidente:

- En primer lugar, se percibe como tendencia necesaria la fusión entre comunicación y marketing, de forma que la comunicación corporativa esté integrada con estrategias de marca y marketing. La integración de comunicación, publicidad y marketing supone para los profesionales de agencia la mejor forma de dar respuesta de modo integral a las necesidades de los clientes, por lo que puede concluirse que el futuro y el reto pasa por una comunicación 360 .

"Los silos de marketing y comunicación se diluyen. El reto es encontrar una orientación política que nos permita adelantarnos al futuro de la comunicación y el marketing y a las necesidades de las organizaciones" (CEO de agencia).

- "Ética, transparencia y expertise continuo" (en declaración textual de otro profesional, CEO de agencia) es otro de los retos de futuro. El trabajo a largo plazo exige a las agencias un nivel de profesionalización y aportación de valor añadido a la comunicación que no se agota en una campaña o servicio puntual, sino que debe funcionar de modo acumulativo en una perspectiva diacrónica, para lograr la confianza de los clientes y de los públicos. Desde el ámbito profesional, se entiende expertise como conocimiento profundo del sector y trayectoria en el mismo.

- En tercer lugar, la capacidad de conectar con los públicos es otro de los retos apuntados. Comunicar directamente al cliente, usuario o empleado "sin intermediación de los medios de comunicación" (comenta otra profesional), lo que implica un reto de "creatividad" y "poner el foco en los contenidos" (ejecutivo de cuentas).

- En cuarto lugar, continúa siendo necesario, desde el punto de vista de las agencias, el reconocimiento del valor de la comunicación por parte de las organizaciones, especialmente de la pyme en España, que según explican todavía concibe la comunicación como un gasto más que como una inversión.

- En quinto lugar, surgen las reflexiones sobre el contexto tecnológico. Aquí se distinguen dos posiciones: las de aquellos más críticos con los cambios tecnológicos, que insisten en que lo importante no es la herramienta o el canal sino la estrategia o el contenido; y las de aquellos que exponen la urgencia de la innovación, transformación digital e integración de aplicaciones digitales, así como una incipiente adaptación permanente a los cambios y un aprendizaje continuado, lo que exige multidisciplinaridad y la colaboración con otros perfiles profesionales.

"En un entorno cada vez más digital y cambiante, las agencias deben estar preparadas para afrontar los grandes retos que sus clientes afrontarán en los próximos años en materia de comunicación: inteligencia artificial, ciber-riesgos, activismo, lucha de las marcas por reclamar la atención de sus consumidores, fake news..., y para ello las agencias deben estar preparadas para responder a estos retos, poniendo siempre las necesidades de los clientes en el centro" (senior HR). 
“Debemos aprender a trabajar con técnicos que nos ayuden a proyectar los principios de la Comunicación” (director de formación).

Sobre la situación del mercado de la comunicación de agencia en España, las visiones de los profesionales son muy diferentes. La mayoría considera que todavía no se ha llegado a un grado de madurez suficiente, especialmente por el reto contextual ("nuevo escenario emergente absolutamente inmaduro", en palabras de un profesional jefe de estrategia) y también atendiendo a cultura de comunicación de las organizaciones, de manera que las pequeñas y medianas empresas todavía no han descubierto y aprehendido el potencial de la comunicación.

"El porcentaje de pymes que cuenta con recursos internos o externos en comunicación es mínimo y lo mismo ocurre con muchas otras organizaciones. Respecto a qué tipo de comunicación se lleva a cabo, salvo las grandes empresas, muchas organizaciones basan su estrategia de posicionamiento y visibilidad en una comunicación todavía dirigida casi en exclusiva a los medios tradicionales" (directora general).

También se apunta la necesidad de una mayor "depuración y concentración de firmas" (vicepresidente de agencia), así como el des-encorsetamiento en unos presupuestos que todavía son considerados limitados. La mayoría considera que el sector evolucionará hacia la especialización.

\subsection{Servicios ofertados y tendencias inmediatas}

A nivel de servicios ofertados se mantiene un extraño equilibrio entre las viejas y nuevas necesidades comunicativas de las organizaciones. A pesar de la transición a nuevos paradigmas y fórmulas comunicativas, las relaciones con los medios siguen siendo consideradas el servicio principal dentro del portafolio de servicios ofertados (por un $78 \%$ de las respuestas), con un descenso de un $11 \%$ con respecto a 2008 (Adecec, 2008).

Le sigue en importancia la comunicación online en redes sociales (65\%) y la elaboración de planes de comunicación (65\%). La organización de eventos y las relaciones públicas continúan siendo consideradas actividades importantes para más de la mitad de las organizaciones participantes. Y para prácticamente la mitad de ellas los servicios de asuntos públicos y comunicación interna resultan asimismo una oferta importante dentro de su cartera. Las campañas con influencers son primordiales para un tercio de las empresas de consultoría.

Importancia menor reciben las actividades de patrocinio, protocolo, publicaciones/editorial y publicidad comercial (producto/marca).

Las que han perdido significación en los últimos diez años son precisamente las consideradas residuales en sus catálogos de servicios actuales, tales como protocolo (muy especialmente), publicaciones, patrocinio y publicidad comercial. Aquellas que han ganado importancia en el último decenio apuntan a la comunicación en las nuevas redes y plataformas (81\%), las campañas con influencers (69\%), la comunicación interna (50\%) y la comunicación audiovisual (47\%).

Datos masivos (big data) (72\%) y comunicación móvil (66\%) son consideradas como las tendencias más destacadas para la profesión en un futuro a corto plazo. Les siguen en importancia las narrativas (47\%), lo que apela a la necesidad de competir por la atención y el tiempo de los públicos en un contexto cada vez más saturado de mensajes. Es en esta tendencia en la que más se encuentran trabajando en la actualidad. También en comunicación móvil y en menor medida en datos masivos. El vídeo en directo es otra tendencia en la que se encuentran trabajando, aunque no es percibida como tan importante a nivel estratégico a medio plazo. Lo mismo sucede con ludificación y vídeo 360.

\subsection{Departamento digital y audiovisual}

En su mayoría (83\%) cuentan con un departamento de comunicación digital, que recibe las siguientes denominaciones: Departamento (de) Digital, Online, Marketing digital, Innovación, Evolution, Digital + Content + Publishing, Social Media Dynamics, Digital Experience, Digital PR. Sus tres funciones más importantes son: gestión de redes sociales, plan de comunicación digital y monitorización y escucha online. A estas tres principales actividades, le sigue desarrollo de campañas online y marketing de contenidos.

Lo digital se ha asentado, por lo tanto, como una necesidad estable de sus estructuras organizativas y de los servicios prestados. Sin embargo, en innovación audiovisual el grado de asentamiento de las necesidades audiovisuales es menor. La mayoría dispone ya de un departamento de creación audiovisual, si bien en un porcentaje inferior a la especialización online. Sube el porcentaje de empresas que externalizan esta función, lo que transmite la idea de una necesidad menos estructural. Las que sí disponen de equipo específico para este tipo de actividades (65\%) emplean las siguientes fórmulas denominativas: PR content, audiovisuales, arte \& innovación, servicios audiovisuales, vídeo, visual content, branded content, y comunicación audiovisual.

A este respecto los principales servicios que ejecutan las agencias son: vídeos para redes sociales, creación de narrativa audiovisual, creación de branded content (contenido para resaltar la marca) y vídeos para YouTube. Se trata por tanto de creación de contenido audiovisual para las nuevas plataformas, fundamentalmente. En menor medida realizan también 
infografías. Y en un tercer plano de importancia, animaciones, spots, vídeos en directo, vídeos 360, marketing audiovisual y vídeos interactivos.

\subsection{Conocimientos de futuro}

Preguntados por los conocimientos de futuro que serán necesarios en los próximos cinco años, se concede mayor importancia a tres específicos vinculados a la comunicación digital, en concreto:

- gestión de contenidos y relaciones en redes sociales,

- desarrollo de aplicaciones móviles, y

- conocimientos de realización audiovisual.

En el extremo contrario, se perciben como de menor interés para el sector los conocimientos de los nuevos profesionales en realidad virtual y en realidad aumentada.

\subsection{Necesidades formativas}

La mayoría de los entrevistados han participado en algún tipo de actividad formativa a lo largo del último año. Reconocen haber invertido entre 1 y 50 horas un $40 \%$ de los encuestados, mientras que un $36 \%$, el doble de tiempo (51 a 100 horas). Entre 101 y 250 horas, un porcentaje muy inferior (13\%). Un 6\% no ha dedicado ningún tiempo a formación.

En cuanto a las necesidades que experimentan de cara a su formación, la profundización de su conocimiento sobre el entorno digital sobresale muy notablemente (65\%). Le siguen en orden de importancia, pero con mucha menor repercusión, las necesidades formativas en liderazgo y capacidades y directivas (25\%), estrategia, marketing y comercialización (21\%) y uso de las

Tabla 3. Conocimientos de futuro

\begin{tabular}{|l|c|}
\hline \multicolumn{1}{|c|}{ Conocimiento } & $\begin{array}{c}\text { Importancia media } \\
\text { percibida (0 a 5) }\end{array}$ \\
\hline Gestión de contenidos y relaciones en redes sociales & 4,37 \\
\hline Aplicaciones móviles & 4,10 \\
\hline Conocimientos de realización audiovisual & 4,00 \\
\hline Publicidad en redes sociales & 3,80 \\
\hline SEO (search engine optimization) & 3,78 \\
\hline Internet de las cosas & 3,60 \\
\hline SEM (search engine marketing) & 3,57 \\
\hline Google Ads (antes Adwords) & 3,12 \\
\hline Realidad virtual & 2,75 \\
\hline Realidad aumentada & 2,58 \\
\hline
\end{tabular}
TICs (21\%).

\subsection{Marca digital}

Además del discurso proyectado por las agencias sobre su situación actual y los retos planteados, la investigación busca un objetivo complementario, el acercamiento a su propia estrategia de comunicación online. Lo que dicen y, en complementariedad, lo que hacen, resultan dos conjuntos de indicadores contrastables y necesarios de la presente investigación. Para ello se ha recurrido al análisis de contenido de sus webs corporativas tal y como se explica en el apartado metodológico.

Los resultados indican que su presencia mínima es en dos redes sociales y la máxima en siete. Las redes sociales preferidas por las agencias españolas son Twitter (donde se encuentra un $85 \%$ de las consultoras) y LinkedIn (en un porcentaje similar). Facebook es la tercera red social en orden de preferencia (70\%) seguida a mayor distancia por Instagram (55\%) y YouTube (45\%). La presencia en la desaparecida Google+, Vimeo, Flickr, Slideshare o Wikipedia es mínima (en torno a un 12\%). De media las agencias de comunicación en España están en 3,4 redes sociales, siendo Twitter y Linkedln las consideradas más importantes para tener proyección en el entorno digital.

En cuanto a los profesionales de agencia, su visibilidad oscila entre el nivel máximo, como es la existencia de webs o blogs personales y el anonimato absoluto, es decir, el de agencias que no dan a conocer el nombre de sus profesionales. Un 27\% de las principales consultoras de comunicación en España apuestan por una opacidad total en este sentido, de modo que no identifican a los miembros de su equipo. En el extremo opuesto, un $10 \%$ de las agencias da visibilidad a las webs o blogs de sus colaboradores.

En lo que respecta a las posibilidades de creación de contenido digital al servicio de su propia estrategia de visibilidad online, 35 (sobre 40) carecen de sala de comunicación online en la que dar a conocer las noticias sobre la organización y en el sector en el que opera. Sin embargo, sí disponen de un espacio de actualidad / novedades la mitad de las consultoras analizadas (20). 17 carecen de blog, mientras que las 23 restantes sí disponen de uno, bien en la web corporativa, bien en otra plataforma pero enlazado desde la web, mediante el que posicionarse como informadores de calidad en el sector de la comunicación y las relaciones con los públicos. Ponen a disposición de los interesados una newsletter o boletín informativo con periodicidad fija un La palabra definitoria del sector en España es transición

total de 13 organizaciones de las 40 analizadas. 
Tabla 4. Recursos de actualidad en las webs corporativas

\begin{tabular}{|c|c|c|c|c|c|c|c|c|}
\hline & \multicolumn{2}{|c|}{ Sala de comunicación } & \multicolumn{2}{|c|}{ Actualidad / noticias } & \multicolumn{2}{|c|}{ Blog } & \multicolumn{2}{|c|}{ Newsletter } \\
\hline & Número & $\%$ & Número & $\%$ & Número & $\%$ & Número & $\%$ \\
\hline Agencias & 5 & 12,5 & 20 & 50 & 23 & 57,5 & 13 & 32,5 \\
\hline
\end{tabular}

Otras opciones de referenciar a la agencia como creadora de branded content y por lo tanto, generadora de artículos, informes, observatorios, aplicaciones o cualquier otra iniciativa que ayude a posicionarla en la arena online, son reducidas aunque reseñables ${ }^{7}$. En concreto:

\section{1) Revistas}

- Revista Uno (Desarrollando Ideas, Llorente \& Cuenca), publicación consultable y descargable, dirigida a clientes, profesionales del sector, periodistas y líderes de opinión, en la que firmas invitadas junto con socios y directivos de la consultora analizan temas relacionados con el mundo de la comunicación.

- Revista Digital Numbers (Kreab). Revista digital consultable online sobre tecnología, economía, finanzas, ciencia, etc.

- Magazine Tinkle (Tinkle). Revista online focalizada en contenido de interés sobre comunicación, estrategias, social media, analítica, marketing y tendencias.

\section{2) Observatorios o centros de generación de conocimiento}

- Desarrollando Ideas (Llorente \& Cuenca). Autodefinido como un "centro de desarrollo de conocimiento", se trata de una iniciativa de la consultora para aportar contenido de interés sobre comunicación, tendencias y contexto tecnológico y socioeconómico. Además de la Revista Uno, editan informes especiales, artículos, un libro con periodicidad anual y estudios focalizados. La iniciativa le ha valido a la consultora diversos premios en certámenes de Comunicación y Relaciones públicas.

- Observatorio de Comunicación Interna (Atrevia). En colaboración con Wolters Kluwer e IE Business Schoool. Localizan y premian las mejores iniciativas de comunicación interna en España. Realizan estudios sobre aspectos que afectan a la comunicación interna, entre ellos, las nuevas plataformas de comunicación para empleados, o los cambios en las relaciones con los públicos internos tanto en la administración pública como en la empresa privada.

- Influencer Hub (Best). Pone en relación influencers y marcas. Ofrece estudios y análisis sobre el sector de la influencia en España.

- Comunidad de Conocimiento (Torres \& Carrera, Grupo Com by Com). Autodefinido como un equipo de comunicación transmedia en el que se integra la consultora Torres \& Carrera, editan periódicamente informes sobre comunicación bajo el cintillo En tiempo de dragones.

- Aula de Liderazgo Público (Mas Consulting) / Observatorio de Asuntos Públicos (Evercom). Entre otras actividades, Mas Consulting incluye una línea de conferencias y publicaciones sobre liderazgo, innovación e inteligencia empresarial. Evercom aborda mediante EverPolitics el análisis de citas electorales destacadas en España y sus repercusiones.

\section{3) Sección de informes, análisis, estudios, white papers, insights}

- Atrevia publica estudios sobre aspectos vinculados a la comunicación y al propio compromiso de la agencia con sus valores, como la igualdad de género.

- Kreab realiza informes sobre el panorama mediático en España o en general sobre el mercado español como oportunidad para invertir. Están por tanto orientados a la consolidación de la agencia en España como polo atractor de capital extranjero.

- Estudio de Comunicación reserva una sección de la web corporativa que incluye: artículos, libros, libros blancos (white papers), análisis sectoriales, estudios de situación política y de caso. Los libros blancos abordan actividades de la comunicación y sus tendencias.

- NewLink publica artículos y noticias, reflexiones sobre comunicación y otros sectores, a los que es posible suscribirse.

- Edelman: barómetros de confianza (sector en el que la marca se ha convertido en referencia a nivel mundial) y estudios orientados a la percepción / conocimiento de los públicos hacia sectores como el entretenimiento o la innovación.

- Mas Consulting: informes de tendencias en comunicación y sobre el contexto político-social en España.

- Apple Tree: informes de comunicación con especial atención al posicionamiento de las marcas en el panorama digital.

- Evercom: informes de tendencias en el sector de la comunicación.

\section{4) Sección de podcasts (Planner Media, Torres \& Carrera)}

La consultora Planner Media mantiene una serie de podcasts de actualidad sanitaria. Torres \& Carrera permite la descarga de podcasts que acompañan algunos de sus análisis / informes.
Perciben los big data como su principal reto de futuro pero mayoritariamente trabajan en narrativa 


\section{5) Canal de televisión (Marco de Comunicación)}

Canal de vídeo consultable en la web corporativa con audiovisuales sobre los trabajos de la agencia, premios recibidos y las perspectivas de sus profesionales.

Los resultados evidencian el blog, el apartado de actualidad y la newsletter como los canales de comunicación con el público general más empleados. Una minoría dispone de otras iniciativas singulares, estructurales y diferenciadas -como se ha relatado- que les permite posicionarse como marca digital de referencia.

El Índice de Liderazgo Digital propuesto en este artículo muestra una radiografía polarizada, en la que conviven consultoras transparentes, con una elevada presencia digital además de creadoras de marca y otras más opacas, menos influyentes y a nivel de imagen más a remolque de los cambios contextuales traídos por la digitalización. Numéricamente la transición se muestra ligeramente inclinada hacia el liderazgo digital.

De promedio, las consultoras obtienen un 6,35 (siendo 15 la máxima puntuación alcanzada) y la moda está en 6. La radiografía permite visualizar un sector en transición, dividido entre un enfoque más tradicional y más innovador casi a partes iguales, con una pequeña cúspide destacada de consultoras innovadoras. Si se tratan los datos sobre la muestra de empresas participantes en la primera parte del estudio, las tendencias no varían.

Tabla 5. Radiografía del sector

\begin{tabular}{|l|c|c|c|c|c|c|}
\cline { 2 - 7 } \multicolumn{1}{c|}{} & \multicolumn{2}{c|}{$\begin{array}{c}\text { Reactividad digital } \\
\text { (1 a 5 puntos) }\end{array}$} & \multicolumn{2}{c|}{$\begin{array}{c}\text { En transición } \\
\text { (6 a 10) }\end{array}$} & \multicolumn{2}{c|}{ Liderazgo digital (11 a 15) } \\
\hline & Número & \% & Número & \% & Número & $\%$ \\
\hline Agencias ranking (40) & 16 & 40,0 & 21 & 52,5 & 3 & 7,5 \\
\hline Agencias muestra (31) & 13 & 41,9 & 15 & 48,4 & 3 & 9,6 \\
\hline
\end{tabular}

Por tipo de consultora, las franquicias de consultoras transnacionales (12) presentan un grado de desarrollo fundamentalmente en transición (75\%, 9 consultoras). De las consultoras de origen local (España), un 42,8\% (12) presenta un desarrollo en transición, frente a un 10,7\% (3) que alcanzan el liderazgo digital y un $46,4 \%$ (13) en un estadio de reactividad digital. Falta por tanto un impulso importante a nivel local.

Atendiendo al tamaño de la consultora, en base a los datos disponibles (sobre 31) el liderazgo digital se corresponde con empresas con mayores recursos humanos o pequeñas consultoras especializadas en comunicación online. En las medianas y pequeñas empresas, el índice de proactividad correlaciona de forma positiva a mayor tamaño según número de profesionales.

\section{Conclusiones}

La palabra definitoria del sector de la consultoría de comunicación en España es transición. Por una parte, debido a que se evoluciona desde las actividades tradicionales hacia otras específicas del entorno digital. Todo ello por medio de la oferta de una estrategia de servicios integrales donde comunicación, publicidad y marketing confluyen en estrategias combinadas para cubrir las necesidades de las organizaciones demandantes de sus servicios. La confluencia y necesaria colaboración entre marketing y comunicación queda patente en el presente estudio y ya había sido sugerida en estudios previos (USC Annenberg Center for Public Relations, 2018; Estanyol-Casals, 2012).

Por otra parte, se muestra la necesidad de seguir trabajando en la concienciación de la importancia de la comunicación para las organizaciones de pequeño y mediano tamaño. Esto refleja colateralmente una doble velocidad de avance, la realidad de la gran empresa y las organizaciones de mayor tamaño y la de las pymes, mucho más ralentizada. Este segundo escenario es percibido por la consultoría de comunicación como de potencial crecimiento si se consigue divulgar una cultura de profesionalización de la comunicación y de sus funciones.

En relación con este escenario de transición se mantiene la conjunción de servicios más tradicionales -como las relaciones con los medios o el plan de comunicación-, con otros más novedosos como la gestión de contenidos en redes sociales o las campañas con influencers. De hecho, el perfil formativo predominante es el de titulado en Periodismo, lo que supone un indicador más del peso de las relaciones informativas en sus carteras de servicios, tal como se lleva apuntando en España desde hace un decenio y coincidiendo con estudios previos y en otros ámbitos geográfico-culturales (Adecec, 2008; Preciado-Hoyos; Nivia-Flórez; Correales-Rivas, 2017; Göncü, 2018). Las estructuras de las agencias reconocen la importancia de la digitalización y por ello demandan en sus profesionales estudios de postgrado o especialización en comunicación y marketing digital. Además, crean departamentos específicos que dan soporte transversal a sus proyectos. Y los propios profesionales participantes en el estudio, a pesar de encontrarse en una media de edad relativamente joven (de 40 a 49 años), reconocen el entorno digital como su necesidad formativa más apremiante, lo que apunta a que los cambios se suceden cada vez más rápido y a la necesidad de estar permanentemente actualizados. 
Todo esto se produce en un contexto de incertidumbre y constante expectativa, en el que no se evidencia una visión clara de hacia dónde se dirige su rol de agencia en un futuro. Sí detectan que una serie de cambios han venido para quedarse: la desintermediación traída por la digitalización y por la web 2.0 y los conocimientos necesarios sobre gestión de comunidades en redes sociales. Más allá de las proyecciones de presente y coincidiendo con estudios a nivel internacional (USC Annenberg Center for Public Relations, 2019) y nacional (Dircom, 2018), big data, creación de contenidos y storytelling se perciben como áreas de futuro y, en el extremo contrario, realidad virtual, realidad aumentada o inteligencia artificial son consideradas tendencias de menor proyección. La comunicación móvil, que ha sido identificada como vector en el que las agencias ya se encuentran trabajando, parece una característica más específica española dada la elevada tasa de penetración de usuarios móviles en España (Ditrendia, 2018).

En todo caso, la formación de los futuros profesionales pasa por la especialización en las nuevas plataformas online. Habrá que seguir observando para comprobar si el perfil de periodismo sigue siendo tan demandado al tiempo que las relaciones con los medios decrecen en importancia. Las facultades y centros de enseñanza deben adaptar sus planes de estudio a la formación en estrategias integrales y multiplataforma, que partan de conocimientos y destrezas transversales como la escucha social y el análisis de datos (Álvarez-Flores; Núñez-Gómez; Olivares-Santamarina, 2018).

Esta situación intermedia, entre viejas y nuevas prácticas, se ve refrendada por su propio desarrollo de marca digital. Las estrategias de liderazgo digital implementadas son sobresalientes en reducidos casos (en concreto, en tres). La marca digital de la agencia debe promoverse en la Red para referenciar a la entidad como proactiva y eficiente en el entorno digital (Costa-Sánchez, 2017; Rodríguez-López; Rodríguez-Álvarez, 2015), además de líder en el sector de la comunicación. Las redes con presencia mayoritaria (Linkedln y Twitter) contrastan con los datos más recientes de empleo de redes sociales en España, que apuntan la caída de Twitter y el estancamiento de Linkedln frente al empuje de Instagram (IAB Spain, 2018).

Se detecta además la falta de una mayor transparencia sobre sus profesionales, lo que contribuiría a singularizar la entidad dándole cara y voz en el contexto digital (Pino; Obispo, 2013). El expertise de la agencia procede en realidad del de sus profesionales. En un entorno en el que se recomienda potenciar la marca personal (Marauri-Castillo; Cantalapiedra-González; Álvarez-Fernández, 2018), convertir la marca agencia en un equipo de destacadas marcas personales puede resultar de interés, a excepción de profesionales que trabajen en temas de lobby y prefieran para su labor el anonimato.

Las posibilidades que les ofrece el nuevo escenario, a nivel de convertirse en think tank de los cambios sociales, sociotecnológicos, relacionales, comunicativos e informativos podría ayudarles en la mejora de su debilidad actual: la falta de proactividad en su visión de futuro y la ausencia de una estrategia destacada de marca digital. Sería de interés además conocer en futuros estudios las repercusiones económicas que están generando los cambios acaecidos en sus estructuras y carteras de servicios.

\section{Notas}

1. En abril de 2018 la agencia Globally fue adquirida por la consultora norteamericana NewLink, aunque sin perder su marca y especializándose en lifestyle.

2. Ketchum y Porter Novelli pasaron a integrarse en Omnicom PRGroup. Se tratan sus datos como Omnicom PRGroup Iberia.

3. La fusión de las agencias Burson Marsteller -que ocupaba la indicada posición 6 en el ranking- y Cohn \& Wolfe dio lugar a la marca Burson Cohn \& Wolfe a nivel mundial, también en España.

4. Perteneciente a Constituency Management Group, empresa que aparece en el informe de PR.

5. Intebrand Branding, a pesar de tratarse de una consultora de marca y no de comunicación en sentido estricto, mantuvo su participación por formar parte del universo propuesto de las agencias de mayor facturación en España.

6. Las categorías resultan una síntesis de la Guía de herramientas tecnológicas para profesionales de la comunicación (Dircom, s/f), Dircom (2017) y USC Annenberg Center for Public Relations (2019).

7. Se excluyen a este respecto las memorias y balances de actividad de la firma.

\section{Referencias}

Aced-Toledano, Cristina (2013). Relaciones públicas 2.0: cómo gestionar la comunicación corporativa en el entorno digital. Barcelona: UOC. ISBN: 9788490292495

Adecec; Dircom (s/f). Manual de buenas prácticas entre consultora y cliente. Madrid: Asociación de Empresas Consultoras en Relaciones Públicas y Comunicación (Adecec); Asociación de Directivos de Comunicación (Dircom).

https://bit.ly/2TMiGaC

Adecec (2008). La comunicación y las relaciones públicas en España. Radiografía de un sector. Madrid: Asociación de Empresas Consultoras en Relaciones Públicas y Comunicación (Adecec). 
https://bit.ly/2HtU5Rf

Alikilic, Ozlem; Atabek, Umit (2012). "Social media adoption among Turkish public relations professionals: A survey of practitioners". Public relations review, v. 38, n. 1, pp. 56-63.

https://doi.org/10.1016/j.pubrev.2011.11.002

Almansa-Martínez, Ana (2004). "Historia de los gabinetes de comunicación en España”. Historia y comunicación social, n. 9, pp. 5-21.

https://revistas.ucm.es/index.php/HICS/article/view/HICS0404110005A

Almansa-Martínez, Ana (2005). "Relaciones públicas y gabinetes de comunicación”. Anàlisi: Quaderns de comunicació $i$ cultura, n. 32, pp. 117-132.

https://core.ac.uk/download/pdf/38977160.pdf

Álvarez-Flores, Erika-Patricia; Núñez-Gómez, Patricia; Olivares-Santamarina, José P. (2018). “Perfiles profesionales y salidas laborales para graduados en publicidad y relaciones públicas: de la especialización a la hibridación”. El profesional de la información, v. 27, n. 1, pp. 136-147.

https://doi.org/10.3145/epi.2018.ene.13

Barquero-Cabrero, José-Daniel (1999). “Evolución cronológica de la profesión de relaciones públicas en España y los aportes de los más importantes expertos". En: Barquero-Cabrero, José-Daniel (coord.). Manual de relaciones públicas empresariales e institucionales. Barcelona: Gestión 2000, pp. 613-664. ISBN: 9788480883283

Buil-Gazol, Pilar; Rodríguez-Salcedo, Natalia (2017). “Las consultoras de comunicación en Cataluña: 25 años de creación de espacios para el diálogo". Doxa comunicación, n. 24, pp. 37-54.

https://recyt.fecyt.es/index.php/doxacom/article/view/58389/35719

Costa-Sánchez, Carmen (2017). "Salas de comunicación online de las entidades bancarias en España: Entre el branded content informativo y el repositorio de notas de prensa”. El profesional de la información, v. 26, n. 4, pp. $744-755$.

https://doi.org/10.3145/epi.2017.jul.18

Dircom (s/f). Guía de herramientas tecnológicas para profesionales de la comunicación. Madrid: Dircom. https://bit.ly/1asuG2U

Dircom (2017). Anuario de la comunicación 2017. Madrid: Dircom.

http://www.dircom.org/anuario-17

Dircom (2018). El estado de la comunicación en España. Madrid: Dircom.

http://www.dircom.org/actualidad-dircom/item/9176-estado-de-la-comunicacion-en-espana-2018

Ditrendia (2018). Mobile en España y en el mundo 2018. Madrid: Ditrendia.

https://mktefa.ditrendia.es/hubfs/Ditrendia-Informe\%20Mobile\%202018.pdf

Estanyol-Casals, Elisenda (2012). "Marketing, public relations and how web 2.0 is changing their relationship: A qualitative asssesment of PR consultancies operating in Spain". Public relations review, v. 38, n. 5, pp. 831-837.

https://doi.org/10.1016/j.pubrev.2012.04.006

Estanyol-Casals, Elisenda; Lalueza-Bosch, Ferran (2014). “¿Tamaño o flexibilidad? Estructura organizativa de las consultoras de relaciones públicas en España". Sphera publica, n. especial, pp. 135-162.

http://sphera.ucam.edu/index.php/sphera-01/article/view/205

Frandsen, Finn; Johansen, Winni; Pang, Augustine (2013). "From management consulting to strategic communication: Studying the roles and functions of communication consulting". International journal of strategic communication, v. 7 , n. 2, pp. 81-83.

https://doi.org/10.1080/1553118X.2013.765439

Göncü, Bârika (2018). "Construction and presentation of communication consultancy expertise: Turkish perspective". Public relations review, v. 44, n. 5, pp. 690-703.

https://doi.org/10.1016/j.pubrev.2018.08.004

Hutton, Michelle (2017). “Our time to lead". In: ICOO; Onepoll (eds). World PR Report 2017, p. 9. https://bit.ly/2HjP8LH

Jenkins, Henry (2008). Convergence culture: La cultura de la convergencia en los medios de comunicación. Barcelona: Paidós. ISBN: 9788449321535

https://stbngtrrz.files.wordpress.com/2012/10/jenkins-henry-convergence-culture.pdf

Marauri-Castillo, Íñigo; Cantalapiedra-González, María-José; Álvarez-Fernández, Carmen (2018). “Blog y Twitter, la combinación perfecta del comunicador digital: los casos de Escolar.net, El comidista y Mi mesa cojea". El profesional de la información, v. 27, n. 2, pp. 349-358. 
https://doi.org/10.3145/epi.2018.mar.13

Martín-Llaguno, Marta; Miquel-Segarra, Susana; Navarro-Beltrá, Marián (2015): “Analysis of communication executives and managers in Spain: Socio-demographic, organizational and attitudinal characteristics from a gender perspective". Catalan journal of communication \& cultural studies, v. 7, n. 2, pp. 129-146.

https://doi.org/10.1386/cjcs.7.2.129_1

Martín-Martín, Fernando (1998). Comunicación en empresas e instituciones. De la consultora a la dirección de comunicación. Salamanca: Ediciones Universidad de Salamanca. ISBN: 9788449321535

Míguez-González, María-Isabel; Costa-Sánchez, Carmen; Túñez-López, Miguel (2019). “Radiografía de las agencias que ofrecen servicios externos de comunicación estratégica y relaciones públicas en regiones periféricas: el caso gallego". Palabra clave, v. 22, n. 1, pp. 92-116.

https://doi.org/10.5294/pacla.2019.22.1.5

Miquel-Segarra, Susana; López-Font, Lorena; Gil-Soldevila, Samuel (2018). “Radiografía de las consultoras de comunicación en España: perfil profesional, estructura y actividad”. Revista latina de comunicación social, n. 73, pp. 478-503. https://doi.org/10.4185/RLCS-2018-1266

Neill, Marlene S.; Schauster, Erin (2018). "Playing nice in the sandbox: Is collaboration among advertising and public relations agencies the same as integration?". Journal of current issues \& research in advertising, v. 39, n. 2, pp. 140-159. https://doi.org/10.1080/10641734.2018.1428248

Pino, Iván; Obispo, María (2013). “Adiós sala de prensa, bienvenida web 2.0. De la prensa de masas a la comunicación en redes". D+i Llorente \& Cuenca, abril.

https://bit.ly/2XYieVI

Preciado-Hoyos, Ángela; Nivia-Flórez, Adriana-Marcela; Correales-Rivas, Juan-Pablo (2017). "The strategic orientation of communications consulting firms in Colombia". Public relations review, v. 43, n. 2, pp. 406-416.

https://doi.org/10.1016/j.pubrev.2017.02.003

PRNoticias; Torres \& Carrera (2018). PR Informe 2018 España-Portugal. Madrid: PRNoticias; Torres \& Carrera. https://bit.ly/2W7ZTEd

Ramírez, Txema (1995). "La influencia de los gabinetes de prensa. Las rutinas periodísticas al servicio del poder". Telos, n. 40, pp. 47-57.

https://telos.fundaciontelefonica.com/archivo/numero040/las-rutinas-periodisticas-al-servicio-del-pode/

Ramírez-Sánchez, Rubén (2018). “Singularidad mediática: hacia una tipología de la post-convergencia más allá de la web 2.0". Intersecciones, v. 1, n. 1, pp. 110-114.

Rodríguez-López, María; Rodríguez-Álvarez, Daniel (2015). “Estudio de la presencia y uso de redes sociales de las agencias de comunicación que más facturan en España”. Revista internacional de relaciones públicas, v. 5, n. 10, pp. 213-236. https://dialnet.unirioja.es/servlet/articulo?codigo $=5306286$

Rodríguez-Salcedo, Natalia; Buil-Gazol, Pilar (2015). "Las agencias de comunicación corporativa en Cataluña". En: Matilla, Kathy (ed.). Historia de la comunicación corporativa en Cataluña. Barcelona: UOC, pp. 205-266. ISBN: 97884 91161547

Rojas-Orduña, Octavio-Isaac (2008). Relaciones públicas: la eficacia de la influencia (2a ed.). Madrid: ESIC. ISBN: 97884 73568777

Röttger, Ulrike; Preusse, Joachim (2013). "External consulting in strategic communication: Functions and roles within systems theory". International journal of strategic communication, v. 7, n. 2, pp. 99-117.

https://doi.org/10.1080/1553118X.2013.765437

Salaverría, Ramón (2003). "Convergencia de los medios”. Chasqui. Revista latinoamericana de comunicación, n. 81, pp. 32-39. https://revistachasqui.org/index.php/chasqui/article/view/1471

Scolari, Carlos A. (2015). Ecología de los medios: entornos, evoluciones e interpretaciones. Barcelona: Gedisa. ISBN: 978 8497848268

Scopen (2017). PR Scope. España 2017.

https://bit.ly/2TQLQFo

Torres \& Carrera (2011). El sector de las relaciones públicas en España. Análisis económico. Torres \& Carrera.

https://bit.ly/2W3q12t

Triantafillidou, Amalia; Yannas, Prodromos (2014). "How public relations agencies in Greece respond to digital trends". Public relations review, v. 40, n. 5, pp. 815-817.

https://doi.org/10.1016/j.pubrev.2014.09.004 
Urzáiz-Fernández-Del-Castillo, Jaime (1997). De las relaciones públicas a la comunicación social integral: nueva estrategia comunicativa para las empresas e instituciones. Madrid: San Martín. ISBN: 9788471403018

USC Annenberg Center for Public Relations (2018). Relevance report. Los Angeles: USC Center for Public Relations. https://annenberg.usc.edu/research/center-public-relations/relevance-report

USC Annenberg Center for Public Relations (2019). Global Communication Report (2019). PR: Tech. The future of technology in communication.

http://assets.uscannenberg.org/docs/2019-global-communications-report.pdf

Von-Platen, Sara (2016). "Struggling with new media and old expertise: reconstructing the professional role of communication consultancy". International journal of strategic communication, v. 10, n. 5, pp. 353-367.

https://doi.org/10.1080/1553118X.2016.1204612

Zerfass, Ansgar; Moreno, Ángeles; Tench, Ralph; Verčič, Dejan; Verhoeven, Piet (2017). European communication monitor 2017. How strategic communication deals with the challenges of visualisation, social bots and hypermodernity. Results of a survey in 50 countries. Brussels: EACD/Euprera, Quadriga Media Berlin. ISBN: 9783942263474

Zerfass, Ansgar; Tench, Ralph; Verhoeven, Piet; Verčič, Dejan; Moreno, Ángeles (2018). European communication monitor 2018. Strategic communication and the challenges of fake news, trust, leadership, work stress and job satisfaction. Results of a survey in 48 countries. Brussels: EACD/Euprera, Quadriga Media Berlin. ISBN: 9783942263504

\section{Anexos}

\subsection{Anexo A. Cuestionario}

1. Datos de contacto
1.1. Agencia
1.2. Nombre
1.3. E-mail
1.4. Teléfono
1.5. Edad

2. Datos agencia
2.1. Año fundación
2.2. Especialización
2.3. Servicios en otros países
2.4. Número de profesionales (H/M)
2.5. Edad media
2.6. Titulación más presente
2.7. Formación de posgrado más demandada

3. Departamentos de la agencia

3.1. Comunicación digital: Denominación y funciones

3.2. Comunicación audiovisual: Denominación y funciones

4. Actividades de comunicación

4.1. Más importantes dentro de su portafolio

4.2. Han ganado relevancia en los últimos 10 años

4.3. Han perdido relevancia en los últimos 10 años

5. Tendencias en comunicación

5.1. Más importantes en los próximos años

5.2. Aquellas que están desarrollando

5.3. Conocimientos necesarios en los próximos cinco años

6. Futuro de la comunicación corporativa y retos como agencia [Libre]

7. Grado de madurez del sector [Libre]

8. Formación

8.1. Tiempo dedicado

8.2. Áreas de mejora 
7.2 Anexo B. Webs y plataformas online

\begin{tabular}{|c|c|c|}
\hline Agencia & Plataforma & URL \\
\hline Apple Tree & Insights & https://www.appletreecommunications.com/es/insights \\
\hline Atrevia & Informes & https://www.atrevia.com/conocimiento \\
\hline Atrevia & Observatorio de Comunicación Interna & https://www.atrevia.com/observatorio-de-la-comunicacion-interna \\
\hline Best & Influencer Hub & https://influencerhub.es \\
\hline Edelman & Insights & https://www.edelman.es/category/insights \\
\hline Evercom & Insights & https://www.evercom.es/insights \\
\hline Evercom & Observatorio de Asuntos Públicos & https://www.evercom.es/asuntos-publicos \\
\hline Llorente \& Cuenca & Revista Uno & https://www.revista-uno.com \\
\hline Llorente \& Cuenca & Desarrollando Ideas & https://ideas.llorenteycuenca.com \\
\hline Kreab & Informes & https://kreab.com/espana/actualidad \\
\hline Kreab & Digital Numbers & https://www.numbersmagazine.com/index.php \\
\hline Estudio de Comunicación & Know-How & https://www.estudiodecomunicacion.com/know-how \\
\hline Marco de Comunicación & $T V$ & https://www.marco.agency/marcotv.php \\
\hline Mas Consulting & Insights & https://www.masconsulting.es/insights \\
\hline Mas Consulting & Aula de Liderazgo & https://www.masconsulting.es/insights/aula-de-liderazgo-publico \\
\hline NewLink & Palabras & http://www.newlink-group.com/es/media \\
\hline Planner Media & Podcasts & $\begin{array}{l}\text { https://www.ivoox.com/podcast-actualidad-sanitaria-140-segundos_sq_ } \\
\text { f166853_1.html }\end{array}$ \\
\hline Tinkle & Magazine Tinkle & https://www.tinkle.es/magazine \\
\hline Torres \& Carrera & Comunidad de Conocimiento & http://corporativo.torresycarrera.com/\#pdfs \\
\hline
\end{tabular}

\section{Inforảrea}

Ayudamos a tu organización en la transformación digital y el gobierno de la información

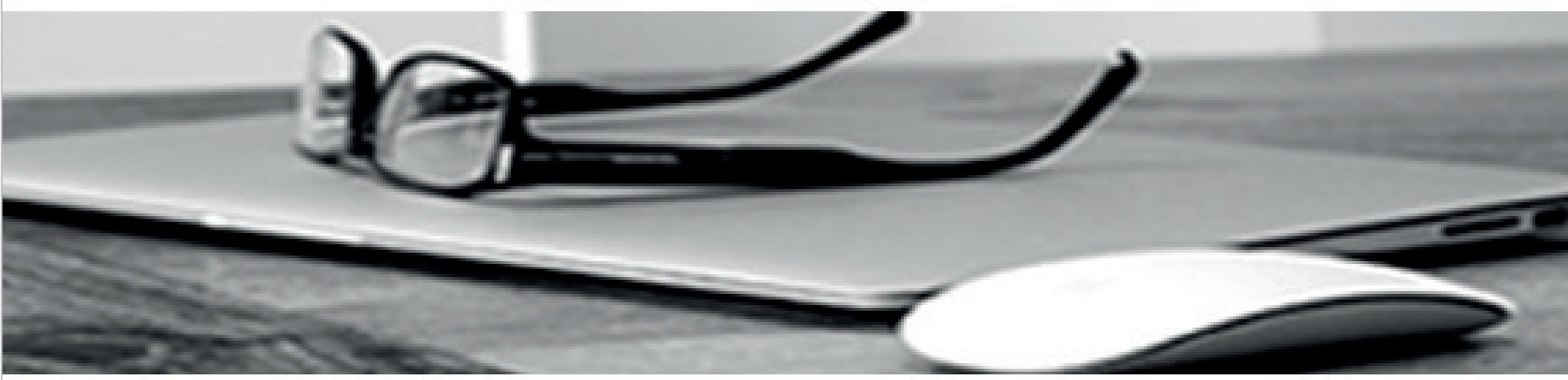

* Consultoría estratégica en gestión y gobierno de la información

* Gestión documental y "records management"

* Gestión de contenidos, intranets corporativas y entornos de colaboración

* Estudios especializados

Clientes satisfechos, cientos de empresas nacionales e internacionales y más de 30 años de experiencia son la mejor garantía de nuestra reputación. 